\title{
On a Class of Integrable Time-Dependent Dynamical Systems
}

\author{
M. V. Bartuccelli** G. Gentile ${ }^{\dagger \dagger}$ \\ * Department of Mathematics and Statistics, University of Surrey, Guildford, GU2 7XH \\ $\dagger$ Dipartimento di Matematica, Università di Roma Tre, Roma, I-00146
}

July 8, 2009

\begin{abstract}
We present some integrable time-dependent systems of classical dynamics, and we apply the results to the equation $\ddot{x}+f(t) x=0$, with $f$ a positive non-decreasing differentiable function; some of the results are extended to the nonlinear case. Moreover we investigate the conditions for the solutions to be bounded and we study their asymptotic behaviour.
\end{abstract}

Keywords Integrable Systems, Time-Dependent Potentials, Boundedness of Solutions

\section{Introduction}

Autonomous Hamiltonian systems with one degree of freedom are completely understood, mainly because their solution curves in phase space are the Hamiltonian contours. When the Hamiltonian depends explicitly upon the time, the dynamics becomes far more complicated, and a general analysis of the motion does not exists.

One aim of the present letter is to construct a class of simple time-dependent systems which are completely integrable, in the sense that they can be solved explicitly in quadratures.

These results are essentially a time-reparametrization; however, to the best of our knowledge, the interesting consequences (see the next section) of this time-reparametrization has never been pointed out clearly in the literature, and another aim of the present letter is to cover this small gap.

We shall then apply the results to the study of systems governed by the equation $\ddot{x}+f(t) x=0$, where $f$ is a positive non-decreasing differentiable function; in particular we shall investigate the conditions for the solutions to be bounded. Partial results for such systems may be found in the literature, for instance, in [12], [3], [11], [13], [9], [10] and [2]. The case of functions $f$ whose derivative can change sign is much more difficult, but it is beyond the intentions of this paper; recent results in this directions have been obtained in [6], [7] and [8]. For a list of related problems of physical relevance we refer, for instance, to [1].

Below, by virtue of the time-reparametrization mentioned above, we define a class of nonconservative systems which naturally extend the classical formula for a conservative system with one degree of freedom, namely Newton's formula

$$
\left\{\begin{array}{l}
\dot{x}(t)=y(t) \\
\dot{y}(t)=-\omega^{2} F(x(t)),
\end{array}\right.
$$

where $\omega \neq 0$ is a constant and $F(x)$ is a $C^{1}$ function of the variable $x$. What happens is that $\omega$ constant case carries over when $\omega=\omega(t)$; in fact we have the following result.

Theorem 1 Given the second order linear time-dependent differential equation

$$
\frac{\mathrm{d}}{\mathrm{d} t}\left(\frac{\dot{x}(t)}{\omega(t)}\right)+\omega(t) x(t)=0
$$

*E.mail: m.bartuccelli@surrey.ac.uk

†E.mail: gentile@mat.uniroma3.it 
where $\omega=\omega(t)$ is a differentiable positive function of time, then its general solution has the form

$$
x(t)=\alpha \cos \left(\int \omega(t) \mathrm{d} t\right) .
$$

Proof. By explicit differentiation.

Remark 1. The expressive formula (3) shows what we anticipated above, namely that the constant coefficient case can be generalised by a natural time-reparametrization.

Remark 2. For the case in which $\omega(t)=1 / t$, the equation reduces to (a particular case of) the well known Euler-Cauchy equation. In fact one has

$$
t^{2} \ddot{x}(t)+t \dot{x}(t)+x(t)=0,
$$

which has the solution $x(t)=\alpha \cos (\ln t+\beta)$.

Now that we have illustrated how to obtain a special generalization of the harmonic oscillator, let us analyze the situation when

$$
\frac{\mathrm{d}}{\mathrm{d} t}\left(\frac{\dot{x}(t)}{\omega(t)}\right)+\omega(t) F(x(t))=0
$$

where $F(x)$ is a nonlinear $C^{1}$ function of $x$. For the nonlinear case it is actually better to use an argument similar to the classical energy equation, which gives the law of conservation of energy. In our context we have the following result. ${ }^{1}$

Theorem 2 The system (5), with $\omega(t)$ a differentiable positive function, has a first integral of the motion given by

$$
H(x(t), \dot{x}(t), t) \equiv \frac{1}{2}\left(\frac{\dot{x}(t)}{\omega(t)}\right)^{2}+U(x(t))=E=\text { constant },
$$

where $U(x)$ is a primitive of $F(x)$.

Proof. We take (5) and multiply through by $\dot{x}(t) / \omega(t)$, obtaining

$$
\frac{\dot{x}(t)}{\omega(t)} \frac{\mathrm{d}}{\mathrm{d} t}\left(\frac{\dot{x}(t)}{\omega(t)}\right)+\dot{x}(t) F(x(t))=0 .
$$

Integrate the above equation to get $(6)$, where $H(x, \dot{x}, t)$ plays the rôle of the total energy of the system and $U(x)$ plays the rôle of the potential energy; hence the theorem is proved.

Multiplying (6) by $\omega^{2}(t)$ and doing some rearrangement we obtain

$$
\int \frac{\mathrm{d} x}{\sqrt{E-U(x)}}= \pm \sqrt{2} \int \mathrm{d} t \omega(t)
$$

Again formula (8) clearly shows that also this result is effectively a time-reparametrization of the time-independent case.

\section{Some applications}

In this section we shall use the results obtained above by virtue of a time-reparametrization and Liapunov's type methods to obtain some results on the dynamical behaviour of the fundamental equation

$$
\ddot{x}(t)+f(t) x(t)=0,
$$

where $f(t)$ is a differentiable function of $t$; if $f(t)>0$ such an equation describes a spring whose elastic constant depends on time. Then the following result holds.

Theorem 3 Suppose that in (9) the function $f(t)$ verify the two conditions: (i) $f(t)>0 \forall t \geq 0$, and (ii) $\dot{f}(t) \geq 0 \forall t \geq 0$. Then both $x(t)$ and $\dot{x}(t) / \sqrt{f(t)}$ remain bounded for all $t \geq 0$.

\footnotetext{
${ }^{1}$ This observation is due to E. I. Dinaburg.
} 
Proof. As $f(t)>0$ we can set $f(t)=\omega^{2}(t)$, with $\omega(t)>0$; one has $\dot{f}(t)=2 \omega(t) \dot{\omega}(t) \geq 0$, so that $\dot{\omega}(t) \geq 0$. Then we can write

$$
\ddot{x}(t)+f(t) x(t)=\ddot{x}(t)+\omega^{2}(t) x(t)=\omega(t)\left[\frac{\mathrm{d}}{\mathrm{d} t}\left(\frac{\dot{x}(t)}{\omega(t)}\right)+\omega(t) x(t)+\frac{\dot{x}(t) \dot{\omega}(t)}{\omega^{2}(t)}\right]=0 .
$$

Define $H(t) \equiv H(x(t), \dot{x}(t), t)$ as

$$
H(t)=\frac{1}{2}\left(\frac{\dot{x}(t)}{\omega(t)}\right)^{2}+\frac{1}{2} x^{2}(t)
$$

then one has

$$
\dot{H}(t)=-\frac{\dot{x}^{2}(t) \dot{\omega}(t)}{\omega^{3}(t)} \leq 0
$$

so that both $x(t)$ and $\dot{x}(t) / \omega(t)$ are bounded by $\sqrt{2 H(0)}$ for all $t \geq 0$.

For the nonlinear case, if we assume that $x=0$ is an isolated minimum point for the potential energy, we have the same result.

Theorem 4 Consider the equation

$$
\ddot{x}(t)+f(t) F(x(t))=0, \quad F(x)=\frac{\mathrm{d} U}{\mathrm{~d} x},
$$

and assume that $x=0$ is an isolated minimum point for the $C^{2}$ function $U(x)$. If $(i) f(t)>0$ $\forall t \geq 0$, and (ii) $\dot{f}(t) \geq 0 \forall t \geq 0$, then for initial data close enough to the origin (in phase space) both $x(t)$ and $\dot{x}(t) / \sqrt{f(t)}$ remain bounded for all $t \geq 0$.

Proof. The proof proceeds exactly as in the linear case: the only (obvious) difference is that one has to use the function (6) instead of (11), and to exploit that $x=0$ is an isolated minimum point for the function $U(x)$.

Remarks.

- Since $\omega(t)$ is positive we can divide $(10)$ by $\omega(t)$ and obtain an equation which differs from (2) by the presence of a "friction" term (that is a term linear in the "velocity" $\dot{x}(t) / \omega(t)$ ): therefore the boundedness of the "energy" is expected, and in fact it has been deduced exactly as in the autonomous case. Therefore the analysis of (10) leading to the theorem 3 follows quite naturally from the results holding for the "frictionless" case dealt with by the theorem 1. Analogous considerations can be drawn about the theorems 2 and 4 .

- If $f(t) \geq 0$ and $\dot{f}(t) \leq 0$, then the boundedness of the solution $(x(t), \dot{x}(t))$ of (13) follows trivially by considering the Lyapunov function

$$
H(t)=\frac{1}{2} \dot{x}^{2}(t)+f(t) U(x(t))
$$

- If we consider the equation

$$
\ddot{x}(t)+p(t) \dot{x}(t)+f(t) F(x(t))=0, \quad F(x)=\frac{\mathrm{d} U}{\mathrm{~d} x},
$$

with $p$ continuous, then it is not difficult to see that the condition for boundedness becomes

$$
2 f(t) p(t)+\dot{f}(t) \geq 0 .
$$

- For $f$ bounded above the theorems are a particular case of [13] and [9]. 


\section{Some examples}

Let us come back to the linear system (9). Suppose as in the statement of the theorem 3 that one has $f(t)>0$ and $\dot{f}(t) \geq 0$ for all $t \geq 0$. By setting $f(t)=\omega^{2}(t)$ and introducing the variable $y(t)=\dot{x}(t) / \omega(t)$, we can write $(9)$ as a time-dependent (linear) planar system

$$
\left\{\begin{array}{l}
\dot{x}(t)=\omega(t) y(t) \\
\dot{y}(t)=-\omega(t) x(t)-\dot{\omega}(t) \omega^{-1}(t) y(t)
\end{array}\right.
$$

if we rescale time by setting

$$
\mathrm{d} \tau=\omega(t) \mathrm{d} t
$$

then we obtain

$$
\left\{\begin{array}{l}
\dot{x}(\tau)=y(\tau) \\
\dot{y}(\tau)=-x(\tau)-\alpha(\tau) y(\tau)
\end{array}\right.
$$

where dots denote derivatives with respect to the arguments, so with respect to $\tau$ in (19), and

$$
\alpha(t)=\frac{\dot{\omega}(t)}{\omega^{2}(t)} \geq 0, \quad \text { so that } \quad \alpha(\tau)=\frac{\dot{\omega}(\tau)}{\omega(\tau)} \geq 0 .
$$

Note that the rescaling of time (18) is well-posed as $\omega(t)>0$ for all $t \geq 0$; of course for any function $t \rightarrow f(t)$ we are setting $f(\tau)=f(t(\tau))$. Note also that the rewriting of $(9)$ as $(17)$ makes more terse the interpretation, stressed in the first remark after the theorem 4 , of the equations of motions as derived from (2) by the introduction of a friction term.

If we pass to polar coordinates, by setting

$$
\left\{\begin{array}{l}
x=\rho \cos \theta \\
y=\rho \sin \theta
\end{array}\right.
$$

then (19) gives

$$
\left\{\begin{array}{l}
\dot{\rho}(\tau)=-\rho(\tau) \alpha(\tau) \sin ^{2} \theta(\tau) \\
\dot{\theta}(\tau)=-1-\alpha(\tau) \sin \theta(\tau) \cos \theta(\tau)
\end{array}\right.
$$

and one has

$$
E(x(\tau), y(\tau)) \equiv H(x(\tau), \dot{x}(\tau), \tau)=\frac{1}{2} y^{2}(\tau)+\frac{1}{2} x^{2}(\tau)=\frac{1}{2} \rho^{2}(\tau) .
$$

The second equation in (22) depends only on $\theta(\tau)$, so that it can be solved, hence used to solve the first one, which at this point, turns out to be a closed time-dependent equation for $\rho(\tau)$.

By defining

$$
A(\tau)=\int_{0}^{\tau} \mathrm{d} \tau^{\prime} \alpha\left(\tau^{\prime}\right), \quad B(\tau)=\int_{0}^{\tau} \mathrm{d} \tau^{\prime} \alpha\left(\tau^{\prime}\right) \sin ^{2} \theta\left(\tau^{\prime}\right),
$$

one obtains, by explicitly integrating the first equation in (22),

$$
\rho(\tau)=\rho(0) \exp [-B(\tau)]
$$

For the system (17) we can state the following result.

Theorem 5 Suppose that in (9) the function $f(t)$ verify the two conditions: (i) $f(t)>0 \forall t \geq 0$, and (ii) $\dot{f}(t) \geq 0 \forall t \geq 0$. Then for the corresponding system (17), as $t \rightarrow \infty$, any solution $(x(t), y(t))$ tends to a limit cycle which is a circle with radius $R \geq 0$. The radius $R$ is strictly positive if and only if $B(\infty)<\infty$.

Proof. As $\dot{E}(\tau) \leq 0$ and $E(\tau) \geq 0$ for all $\tau \geq 0$, there exists the limit

$$
\lim _{\tau \rightarrow \infty} E(\tau)=E_{0} \geq 0
$$

which defines a curve

$$
\gamma=\left\{(x, y) \in \mathbb{R}^{2}: E(x, y)=E_{0}\right\}
$$

such a curve is a circle with radius $R=\rho(\infty)$, and it is easy to show that such a curve is the $\omega$-limit set of the solution with initial datum $(x(0), y(0))$ : therefore it is a limit cycle [5]. If $B(\infty)<\infty$ then $\rho(\infty)>0$, while if $B(\infty)=\infty$ then $\rho(\infty)=0$. 
Remark. Of course when $R=0$ in the theorem 5 , the corresponding limit circle degenerates into the equilibrium point $x=0$. As the theorem 3 implies that $x=0$ is a stable equilibrium point, we can conclude that in such a case $x=0$ is asymptotically stable.

As concrete examples let us consider the two cases $(1) \omega(t)=1+t^{2}$, and $(2) \omega(t)=\left(1+2 t^{2}\right) /(2+$ $\left.2 t^{2}\right)$; in both cases $\omega(t)>0$ and $\dot{\omega}(t)>0$ for $t \geq 0$, while $\omega(t)$ diverges in the first case and tends to 1 in the second case.

In the first case one has from (20)

$$
\alpha(t)=\frac{2 t}{\left(1+t^{2}\right)^{2}}
$$

so that $\alpha(\tau)$ behaves as $O(1 / \tau)$ for large $\tau$. Therefore $A(\infty)=\infty$, and one can show (see Appendix) that also $B(\tau)$ diverges for $\tau \rightarrow \infty$. This means that all solution are asymptotic to the origin, which turns out to be an asymptotically stable equilibrium point (see the above remark).

In the second case one has from (20)

$$
\alpha(t)=\frac{4 t}{\left(1+2 t^{2}\right)^{2}}
$$

so that $\alpha(\tau)$ behaves as $O\left(1 / \tau^{3}\right)$ for large $\tau$. Therefore $A(\infty)<\infty$, and, as $B(\tau) \leq A(\tau)$, we see that also $B(\infty)$ is finite. In such a case we can apply the theorem 5 and conclude that there is a limit cycle with strictly positive radius.

As another application let us consider a pendulum with variable length $\ell(t)$, according to some prescribed law; the corresponding Lagrangian is (we take the mass $m=1$ and gravity $g=1$ )

$$
\mathcal{L}(\theta, \dot{\theta}, t)=\frac{1}{2}\left(\dot{\ell}^{2}(t)+\ell^{2}(t) \dot{\theta}^{2}\right)+\ell(t)(\cos \theta-1)
$$

so that the Euler-Lagrange equation for the variable $\theta$ is given by

$$
\ell(t) \ddot{\theta}(t)+2 \dot{\ell}(t) \dot{\theta}(t)+\sin \theta(t)=0 ;
$$

let us define $\ell(t)=1 / \omega^{2}(t)$, then equation (31) becomes

$$
\frac{\ddot{\theta}(t)}{\omega^{2}(t)}-4 \frac{\dot{\theta}(t) \dot{\omega}(t)}{\omega^{3}(t)}+\sin \theta(t)=0
$$

By defining

$$
H(t) \equiv H(\theta(t), \dot{\theta}(t), t)=\frac{1}{2}\left(\frac{\dot{\theta}(t)}{\omega(t)}\right)^{2}+(1-\cos \theta(t)),
$$

and by using that

$$
\begin{aligned}
\frac{1}{2} \frac{\mathrm{d}}{\mathrm{d} t}\left(\frac{\dot{\theta}(t)}{\omega(t)}\right)^{2} & =\left(\frac{\dot{\theta}(t)}{\omega(t)}\right)\left[\frac{\ddot{\theta}(t)}{\omega(t)}-\frac{\dot{\theta}(t) \dot{\omega}(t)}{\omega^{2}(t)}\right] \\
& =\dot{\theta}(t)\left[\frac{\ddot{\theta}(t)}{\omega^{2}(t)}-\frac{\dot{\theta}(t) \dot{\omega}(t)}{\omega^{3}(t)}\right]=\dot{\theta}(t)\left[-\sin \theta(t)+3 \frac{\dot{\theta}(t) \dot{\omega}(t)}{\omega^{3}(t)}\right],
\end{aligned}
$$

one can see that

$$
\dot{H}(t)=\frac{3 \dot{\theta}^{2}(t) \dot{\omega}(t)}{\omega^{3}(t)} .
$$

In the linear approximation (31) becomes

$$
\ell(t) \ddot{\theta}(t)+2 \dot{\ell}(t) \dot{\theta}(t)+\theta(t)=0,
$$

which, expressed in terms of $\omega(t)$ gives

$$
\frac{\ddot{\theta}(t)}{\omega^{2}(t)}-4 \frac{\dot{\theta}(t) \dot{\omega}(t)}{\omega^{3}(t)}+\theta(t)=0
$$


for such an equation we can define $H(t)$ as

$$
H(t) \equiv H(\theta(t), \dot{\theta}(t), t)=\frac{1}{2}\left(\frac{\dot{\theta}(t)}{\omega(t)}\right)^{2}+\frac{1}{2} \theta^{2}(t),
$$

and one sees that (35) is satisfied for the function (38) along the flow of (36).

Then we can state the following result about the linearized equation of the pendulum with length increasing in time.

Theorem 6 Consider the system described by the equation (36) and suppose that one has $\dot{\ell}(t)>0$ (physically it is a linearized pendulum with strictly increasing length). All motions remain bounded; furthermore if one makes the extra assumption that $\dot{\ell}(t) / \ell(t)$ is not summable at infinity, such a solution evolves to zero.

Proof. Write (36) in the form (37). As $\dot{\ell}(t)>0$ then $\dot{\omega}(t)<0$, so that as a consequence of (35) we have that $\dot{H}(t)<0$. Therefore we can reason as in the proof of the theorems 3 and 4 , and conclude that $(\theta(t), \dot{\theta}(t) / \omega(t))$ remains bounded.

By setting $y(t)=\theta(t) / \dot{\omega}(t)$, we obtain from $(37)$

$$
\left\{\begin{array}{l}
\dot{\theta}(t)=\omega(t) y(t) \\
\dot{y}(t)=-\omega(t) \theta(t)+3 \dot{\omega}(t) \omega^{-1}(t) y(t)
\end{array}\right.
$$

which, after rescaling time through (18), can be written in the form (19), with $(x(\tau), y(\tau))=$ $(\theta(t(\tau)), y(t(\tau)))$ and $\alpha(\tau)=\alpha(t(\tau))$, where

$$
\alpha(t)=-3 \frac{\dot{\omega}(t)}{\omega^{2}(t)}=\frac{3 \dot{\ell}(t)}{2 \sqrt{\ell(t)}} \geq 0 .
$$

Under the hypothesis that $\dot{\ell}(t) / \ell(t)$ is not summable at infinity, one has that

$$
A(\tau)=\int_{0}^{\tau} \mathrm{d} \tau^{\prime} \alpha\left(\tau^{\prime}\right)=\int_{0}^{t} \mathrm{~d} t^{\prime} \alpha\left(t^{\prime}\right) \omega\left(t^{\prime}\right)=\frac{3}{2} \int_{0}^{t} \mathrm{~d} t^{\prime} \frac{\dot{\ell}\left(t^{\prime}\right)}{\ell\left(t^{\prime}\right)}
$$

diverges as $\tau \rightarrow \infty$, and we can show that the same holds also for $B(\tau)$ (see Appendix), so that one can conclude that $\rho(\infty)=0$; then by the theorem 5 the origin is asymptotically stable.

Remark. As a particular case of the theorem 6 we can consider $\ell(t)=t^{2}$; in such a case the linearization of (31) reduces to Euler-Cauchy equation

$$
t^{2} \ddot{\theta}(t)+4 t \dot{\theta}(t)+\theta(t)=0,
$$

which can be explicitly solved, giving $\theta(t)=a t^{\gamma_{+}}+b t^{\gamma_{-}}$, with $\gamma_{ \pm}=(-3 \pm \sqrt{5}) / 2$ and $a, b$ constants depending on the initial datum. Therefore the theorem 6 can be seen as a generalization of such a well known result.

\section{A Appendix}

Consider the system (19) with $\alpha(t)$ given by (28); one has for the angle variable

$$
-1-\alpha(\tau) \leq \dot{\theta}(\tau) \leq-1+\alpha(\tau),
$$

so that, by defining $\varphi(\tau)=-\theta(\tau)$, one has

$$
1-\alpha(\tau) \leq \dot{\varphi}(\tau) \leq 1+\alpha(\tau)
$$

since $\alpha(\tau)=O(1 / \tau)$ for $\tau \rightarrow \infty$, we can deduce that, for $\tau$ large enough, say $\tau \geq T$ for some large $T$, the function $\varphi(\tau)$ is increasing in time at a rate bounded from below and from above, say $1 / 2<\dot{\varphi}(\tau)<2$.

In terms of $\varphi(\tau)$ the function $B(\tau)$ becomes

$$
B(\tau)=\int_{0}^{\tau} \mathrm{d} \tau^{\prime} \alpha\left(\tau^{\prime}\right) \sin ^{2} \varphi\left(\tau^{\prime}\right) ;
$$


the integrand appearing in (45) behaves like a constant times $\sin ^{2} \varphi\left(\tau^{\prime}\right) / \tau^{\prime}$ for $\tau^{\prime} \geq T$.

Call $\tau_{k}, k \in \mathbb{N}$, the (ordered) values of $\tau \geq T$ at which $\sin ^{2} \varphi(\tau)$ vanishes and set $\Delta \tau_{k}=\tau_{k+1}-\tau_{k}$. One has

$$
\pi=\varphi\left(\tau_{k+1}\right)-\varphi\left(\tau_{k}\right)=\int_{\tau_{k}}^{\tau_{k+1}} \mathrm{~d} \tau^{\prime} \dot{\varphi}\left(\tau^{\prime}\right) \geq\left|\dot{\varphi}\left(\tau_{k}\right)\right| \Delta \tau_{k},
$$

so that

$$
\Delta \tau_{k} \leq \frac{\pi}{\dot{\varphi}\left(\tau_{k}\right)} \leq 2 \pi \quad \text { hence } \quad \tau_{k+1} \leq \tau_{1}+2 \pi k .
$$

Then we can reason as follows in order to show that one has $B(\infty)=\infty$. We can split the integral (45) defining $B(\infty)$ into two parts, the first from 0 to $\tau_{1}$ and the second from $\tau_{1}$ to $\infty$. The second integral can be bounded from below by a constant times

$$
\begin{aligned}
\sum_{k=1}^{\infty} \int_{\tau_{k}}^{\tau_{k+1}} \mathrm{~d} \tau^{\prime} \frac{\sin ^{2} \varphi\left(\tau^{\prime}\right)}{\tau^{\prime}} & \geq \sum_{k=1}^{\infty} \frac{1}{\tau_{k+1}} \int_{\tau_{k}}^{\tau_{k+1}} \mathrm{~d} \tau^{\prime} \sin ^{2} \varphi\left(\tau^{\prime}\right) \\
& \geq \sum_{k=1}^{\infty} \frac{1}{\tau_{k+1}} \int_{\pi\left(n_{0}+k\right)}^{\pi\left(n_{0}+k+1\right)} \mathrm{d} \varphi \frac{1}{\left|\dot{\varphi}\left(\tau^{\prime}\right)\right|} \sin ^{2} \varphi \\
& \geq \sum_{k=1}^{\infty} \frac{1}{2 \tau_{k+1}} \int_{\pi\left(n_{0}+k\right)}^{\pi\left(n_{0}+k+1\right)} \mathrm{d} \varphi \sin ^{2} \varphi \geq \sum_{k=k_{0}}^{\infty} \frac{C}{\tau_{1}+2 \pi k}
\end{aligned}
$$

where $n_{0}$ is an integer depending on $\tau_{1}$ and $C / 2$ is the value of the integral of $\sin ^{2} \theta$ over the interval $[0, \pi]$. The series in (48) is obviously diverging (as it is a harmonic series), so that we can conclude $B(\infty)=\infty$.

Remark. Note that in proving the divergence of $B(\infty)$ what is really needed is not the exact form of $\alpha(t)$, but only the fact that $\alpha(\tau)$ goes to zero at infinity in a not summable way. In fact under such a weaker assumption in (48) one obtains $\alpha\left(\tau_{k+1}\right)$ instead of $1 / \tau_{k+1}$, and the series still diverges as still (47) holds and, for any positive function $f(\tau)$ and for any diverging increasing sequence $\left\{\tau_{k}\right\}$, such that $\Delta \tau_{k}=\tau_{k+1}-\tau_{k}$ is uniformly bounded, one has

$$
C^{-1} \sum_{k=1}^{\infty} f\left(\tau_{k}\right)<\int_{\tau_{1}}^{\infty} \mathrm{d} \tau f(\tau)<C \sum_{k=1}^{\infty} f\left(\tau_{k}\right),
$$

for a suitable constant $C$, so that the series diverges if the function is not summable. Hence the above discussion can be used in order to prove that one has $B(\infty)=\infty$ also in the case of the theorem 6 , if $\dot{\ell}(t) / \ell(t)$ tends to zero in a not summable way.

If under the hypotheses of the theorem 6 the quantity $\dot{\ell}(t) / \ell(t)$, hence $\alpha(\tau)$, does not tend to zero, then one can reason as above with $\alpha\left(\tau_{k+1}\right)$ replaced with $\max _{\tau \in\left[\tau_{k}, \tau_{k+1}\right]} \alpha(\tau)$, and the same conclusions hold: this means that $B(\infty)=\infty$ also in such a case.

\section{Acknowledgments}

We thank Efim I. Dinaburg for useful and stimulating discussions.

\section{References}

[1] V. I. Arnol'd, Ordinary Differential Equations, Springer, Berlin, 1992.

[2] M. Cecchi, M. Marini, G. Villari, On some classes of continuable solutions of a nonlinear differential equation, J. Differential Equations 118 (1995), no. 2, 403-419.

[3] L. Cesari, Asymptotic behavior and stability problems in ordinary differential equations, Third edition, Ergebnisse der Mathematik und ihrer Grenzgebiete, Band 16, Springer-Verlag, New York-Heidelberg, 1971 (First edition 1959).

[4] W. A. Coppel, Stability and asymptotic behavior of differential equations, D. C. Heath and Co., Boston, 1965. 
[5] M. W. Hirsch \& S. Smale, Differential Equations, Dynamical Systems, and Linear Algebra, Academic Press, New York, 1974.

[6] S. Laederich \& M. Levi, Invariant curves and time-dependent potentials, Ergodic Theory Dynam. Systems 11 (1991), no. 2, 365-378.

[7] M. Levi, Quasiperiodic motions in superquadratic time-periodic potentials, Comm. Math. Phys. 143 (1991), no. 1, 43-83.

[8] M. Bartuccelli, J. Deane, G. Gentile, S. Gourley, Global Attraction to the Origin in a Parametrically-Driven Nonlinear Oscillator, Submitted.

[9] M. Marini, Criteri di limitatezza per le soluzioni dell'equazione lineare del secondo ordine, Boll. Un. Mat. Ital. (4) 11 (1975), no. 1, 154-165.

[10] M. Marini, P. Zezza, On the asymptotic behavior of the solutions of a class of second-order linear differential equations, J. Differential Equations 28 (1978), no. 1, 1-17.

[11] V. V. Nemytskii, V. V. Stepanov, Qualitative theory of differential equations, Princeton Mathematical Series No. 22, Princeton University Press, Princeton, 1960.

[12] G. Sansone, Equazioni differenziali nel campo reale, Vol. II, Zanichelli, Bologna, 1941.

[13] G. Trevisan, Un teorema di stabilità per le equazioni differenziali lineari del secondo ordine, Atti Accad. Naz. Lincei. Rend. Cl. Sci. Fis. Mat. Nat. (8) 28 (1960), 467-469. 